\title{
Hands-on Analysis of 802.11ac Modulation and Coding Scheme
}

\author{
Doru Gabriel Balan, Alin Dan Potorac, Radu Cezar Tărăbuță \\ Ştefan cel Mare University of Suceava / Computers, Electronics and Automation Department, Suceava, Romania \\ Email address: \\ dorub@usv.ro (D. G. Balan), alinp@eed.usv.ro (A. D. Potorac), radut@stud.usv.ro (R. C. Tărăbuță)
}

To cite this article:

Doru Gabriel Balan, Alin Dan Potorac, Radu Cezar Tărăbuță. Hands-on Analysis of 802.11AC Modulation and Coding Scheme. International Journal of Wireless Communications and Mobile Computing. Vol. 3, No. 5, 2015, pp. 46-50. doi: 10.11648/j.wcmc.20150305.11

\begin{abstract}
Current communication networks are full of mobile devices that are capable of performing data transfers at high data rates or access high resolution video streams. For such requirements, in the data communications there is a permanent concern of wireless communications development to keep up with the wired communication networks. Because the $802.11 \mathrm{n}$ technology is not fast enough for increasingly more users willing games and online broadcasts, the 802.11ac technology was developed. This new IEEE standard, along with the future technology $802.11 \mathrm{ad}$, is aiming to achieve a new level of performance, called VHT (Very High Throughput). The goal is to reach transfer rates (over 1Gbps for now) comparable to those establish in wired networks. This article is proposing a study over 802.11 ac technology by exploring the performances of the specific MCS (Modulation and Coding Scheme) with a handy digitally modulated signals investigation method, CCDF (Complementary Cumulative Distribution Function).
\end{abstract}

Keywords: Wireless, 802.11ac, Modulation, Coding Scheme

\section{Introduction}

IEEE $802.11 \mathrm{ac}[1]$ is one of the ongoing WLAN standards aiming to support very high throughput (VHT) with data rate of up to $6 \mathrm{Gbps}$ below the $6 \mathrm{GHz}$ band. [2].

The increasing demands on modern Wi-Fi networks are based on several trends that are requiring ever greater levels of performance, scalability, and availability [3]:

- Proliferation of mobile devices (devices as smartphones and tablets rely exclusively on Wi-Fi for network connectivity);

- Multiple devices per user (most users are using concurrently multiple devices with networking capabilities.);

- Always-on connectivity (it is common that today users have permanent connectivity to the Internet);

- Wi-Fi as the primary network access method;

- IoT (Internet of Things), IoE (Internet of Everything) and BYOD (Bring Your Own Device) expansion;

- Cellular network offload (3G, 4G and future 5G network protocols are avoiding network congestion);

- Hotspots are becoming increasingly more important.

- The theoretical maximum data rate and actual user throughput, that will be lower due to the shared medium, and transmission, management and control overhead, depend on a number of factors, such as:

- Physical-layer (PHY) connection rates;

- Number of spatial streams;

- Allocated channel width;

- Modulation and coding scheme (MCS) used;

- Guard interval (time between transmitted characters).

\section{Wireless Technology Elements}

\subsection{11ac Characteristics}

From this perspective, several characteristics of $802.11 \mathrm{ac}$ technology can be easily identified $[4,5]$, such as:

- Introduces the new VHT operating mode, that is a mixt mode, supporting 802.11a and 802.11n clients in the 5 Ghz band;

- Supports from 1 to 8 (up to 4 per client) spatial streams between infrastructure access points and wireless clients, using several principles, like:

- Space multiplexing (signal is splinted in multiple signal streams, each transmitted in a separate spatial stream),

- Space Time Block Coding - STBC (uses more antennas to redundantly transmit a single traffic 
stream over multiple RF paths),

- Multi-User Multiple Input Output (MU-MIMO) use a multiple antenna array to direct beams and transmit simultaneously to multiple clients.

- Wider RF transmission channels, it adds $80 \mathrm{MHz}$ and $160 \mathrm{MHz}$ channels to the $5 \mathrm{GHz}$ band;

- Higher Modulation and Coding Schemes (MCS), introducing the 256 QAM signal modulation;

- Specifies 10 MCS indices (0-9, as in Figure 1);

When all specified enhancements are enabled, 802.11ac delivers a maximum PHY data rate of $6933 \mathrm{Mbps}$, as can be seen in Figure 1, in the last filled cell.

\subsection{Specific MCS - Modulation and Coding Scheme}

As seen before, network communications via 802.11ac can be realized using one of the $10 \mathrm{MCS}$ provided with maximum 8 spatial streams. Figure 1 is presenting the $802.11 \mathrm{ac}$ characteristics, regarding PHY data rates and MCS, for only two situations of user number of spatial streams, namely for 1 spatial stream and for 8 spatial streams. Obviously there are also data for the other cases, (the cases with 2-7 spatial streams), which were excluded from organizing considerations.

\begin{tabular}{|c|c|c|c|c|c|c|c|c|c|c|c|}
\hline \multirow[b]{2}{*}{ MCS } & \multirow[b]{2}{*}{ Modulation } & \multirow{2}{*}{$\begin{array}{l}\text { Bits per } \\
\text { Symbol }\end{array}$} & \multirow{2}{*}{$\begin{array}{c}\text { Coding } \\
\text { Ratio }\end{array}$} & \multicolumn{2}{|c|}{ 20-MHz } & \multicolumn{2}{|c|}{$40 \cdot \mathrm{MHz}$} & \multicolumn{2}{|c|}{$80 \cdot \mathrm{MHz}$} & \multicolumn{2}{|c|}{$160-\mathrm{MHz}$} \\
\hline & & & & 800 ns & $400 \mathrm{~ns}$ & 800 ns & $400 \mathrm{~ns}$ & 800ns & $400 \mathrm{~ns}$ & $800 \mathrm{~ns}$ & $400 \mathrm{~ns}$ \\
\hline \multicolumn{4}{|c|}{1 Spatial Stream } & \multicolumn{8}{|c|}{ Data Rate (Mbps) } \\
\hline MCSO & BPSK & 1 & $1 / 2$ & 6.5 & 7.2 & 13.5 & 15.0 & 29.3 & 32.5 & 58.5 & 65.0 \\
\hline MCS 1 & QPSK & 2 & $1 / 2$ & 13.0 & 14.4 & 27.0 & 30.0 & 58.5 & 65.0 & 117.0 & 130.0 \\
\hline $\operatorname{MCS} 2$ & QPSK & 2 & $3 / 4$ & 19.5 & 21.7 & 40.5 & 45.0 & 87.8 & 97.5 & 175.5 & 195.0 \\
\hline $\operatorname{MCS} 3$ & 16-QAM & 4 & $1 / 2$ & 26.0 & 28.9 & 54.0 & 60.0 & 117.0 & 130.0 & 234.0 & 260.0 \\
\hline $\operatorname{MCS} 4$ & 16-QAM & 4 & $3 / 4$ & 39.0 & 43.3 & 81.0 & 90.0 & 175.5 & 195.0 & 351.0 & 390.0 \\
\hline $\operatorname{MCS} 5$ & 64-QAM & 6 & $2 / 3$ & 52.0 & 57.8 & 108.0 & 120.0 & 234.0 & 260.0 & 468.0 & 520.0 \\
\hline $\operatorname{MCS} 6$ & 64-QAM & 6 & $3 / 4$ & 58.5 & 65.0 & 121.5 & 135.0 & 263.3 & 292.5 & 526.5 & 585.0 \\
\hline MCS 7 & 64-QAM & 6 & $5 / 6$ & 65.0 & 72.2 & 135.0 & 150.0 & 292.5 & 325.0 & 585.0 & 650.0 \\
\hline MCS 8 & 256-QAM & 8 & $3 / 4$ & 78.0 & 86.7 & 162.0 & 180.0 & 351.0 & 390.0 & 702.0 & 780.0 \\
\hline $\operatorname{mCs} 9$ & 256-QAM & 8 & $5 / 6$ & $N / A$ & N/A & 180.0 & 200.0 & 390.0 & 433.3 & 780.0 & 866.7 \\
\hline \multicolumn{4}{|c|}{8 Spatial Streams } & \multicolumn{8}{|c|}{ Data Rate (Mbps) } \\
\hline MCSO & BPSK & 1 & $1 / 2$ & 52.0 & 57.8 & 108.0 & 120.0 & 234.0 & 260.0 & 468.0 & 520.0 \\
\hline MCS1 & QPSK & 2 & $1 / 2$ & 104.0 & 115.6 & 216.0 & 240.0 & 468.0 & 520.0 & 936.0 & 1040.0 \\
\hline MCS2 & QPSK & 2 & $3 / 4$ & 156.0 & 173.3 & 324.0 & 360.0 & 702.0 & 780.0 & 1404.0 & 1560.0 \\
\hline $\operatorname{MCS} 3$ & 16-QAM & 4 & $1 / 2$ & 208.0 & 231.1 & 432.0 & 480.0 & 936.0 & 1040.0 & 1872.0 & 2080.0 \\
\hline $\operatorname{MCS} 4$ & 16.QAM & 4 & $3 / 4$ & 312.0 & 346.7 & 648.0 & 720.0 & 1404.0 & 1560.0 & 2808.0 & 3120.0 \\
\hline MCS 5 & 64-QAM & 6 & $2 / 3$ & 416.0 & 462.2 & 864.0 & 960.0 & 1872.0 & 2080.0 & 3744.0 & 4160.0 \\
\hline MCS 6 & 64-QAM & 6 & $3 / 4$ & 468.0 & 520.0 & 972.0 & 1080.0 & 2106.0 & 2340.0 & 4212.0 & 4680.0 \\
\hline MCS 7 & 64-QAM & 6 & $5 / 6$ & 520.0 & 577.8 & 1080.0 & 1200.0 & 2340.0 & 2600.0 & 4680.0 & 5200.0 \\
\hline MCS 8 & 256-QAM & 8 & $3 / 4$ & 624.0 & 693.3 & 1296.0 & 1440.0 & 2808.0 & 3120.0 & 5616.0 & 6240.0 \\
\hline $\operatorname{MCS} 9$ & 256-QAM & 8 & $5 / 6$ & $N / A$ & $N / A$ & 1440.0 & 1600.0 & 3120.0 & 3466.7 & 6240.0 & 6933.3 \\
\hline
\end{tabular}

Figure 1. 802.11ac PHY data rates for 1 and 8 spatial streams [3].

Figure 2 shows the current state of technology development 802.11 ac identified by the initially developed set of $802.11 \mathrm{ac}$ equipment (802.11ac 1st wave). As can be seen, the actual data rate is $1300 \mathrm{Mbps}$.

In the results included in this article we have concentrated over 802.11ac specific Modulation and Coding Schemes (MCS). Our determinations are based on the CCDF (Complementary Cumulative Distribution Function) investigation method.

\begin{tabular}{|c|c|c|c|c|}
\hline $\begin{array}{c}\text { Channel Width } \\
\left(\mathrm{MHz}^{2}\right)\end{array}$ & Spatial Streams & $\begin{array}{l}\text { 802.11ac MCS } \\
\text { Index }\end{array}$ & Guard Interval & $\begin{array}{c}\text { PHY Data Rate } \\
\text { (Mbos) }\end{array}$ \\
\hline 20 & 1 & 5 & โG & 52 \\
\hline 40 & 4 & 7 & SGI & 600 \\
\hline 40 & 4 & 9 & SGI & 800 \\
\hline 80 & 1 & 9 & SGI & 433 \\
\hline 80 & 2 & 9 & SGI & 867 \\
\hline 80 & 3 & 9 & SGI & 1300 \\
\hline 80 & 4 & 9 & SGI & 1733 \\
\hline 80 & 8 & 9 & $S G \mid$ & 3467 \\
\hline 160 & 1 & 9 & $S G I$ & 867 \\
\hline 160 & 2 & 9 & SGI & 1733 \\
\hline 160 & 3 & 8 & SGI & 2340 \\
\hline 160 & 4 & 9 & $S G I$ & 3467 \\
\hline 160 & 8 & 9 & SGI & 6933 \\
\hline
\end{tabular}

Figure 2. 802.11 PHY data rates in real use [6].

\section{Investigation Method}

Measurement of the CCDF is often used for evaluating nonlinearities of amplifiers or transmitter output stages, for instance. This measurement indicates how often the observed signal reaches or exceeds a specific level. From a physical point of view, the CCDF measurement is the integral of the distribution function versus the level (integration of the observed level to infinity).

Comparison of evaluated or measured values and theoretical reference values (as CCDF Gaussian), as it can be seen in Figures 5-14, quickly yields information on the nonlinear response of all types of active elements. However, the great advantage of the CCDF measurement is that the useful signal itself is analyzed; as a result, it is not necessary to transmit complex test sequences. CCDF determination is a very important measurement in RF transmission systems. Measuring the CCDF is a simple and effective method of determining the nonlinear characteristics of active elements. [6].

\section{Testbed Elements}

To achieve the aimed performance analysis on 802.11ac modulation and coding scheme, it was used a testing space consisting of a Keysight (formerly Agilent) set of tools.

There are two key elements of the test bench that provided the results presented in this paper:

The first element (software) is the N7617B Signal Studio for WLAN 802.11a/b/g/n/ac [7], used to design the 802.11 ac signals;

The second element (hardware) is a vector signal generator, N5182A MXG [8], with N7617B option, for testing loaded signals from software.

The interconnection of these components, as in Figure 3, allows controlling the theoretical elements for $802.11 \mathrm{ac}$ technology-specific signals description and the signal characteristics validation, achieved through vector signal generator. 


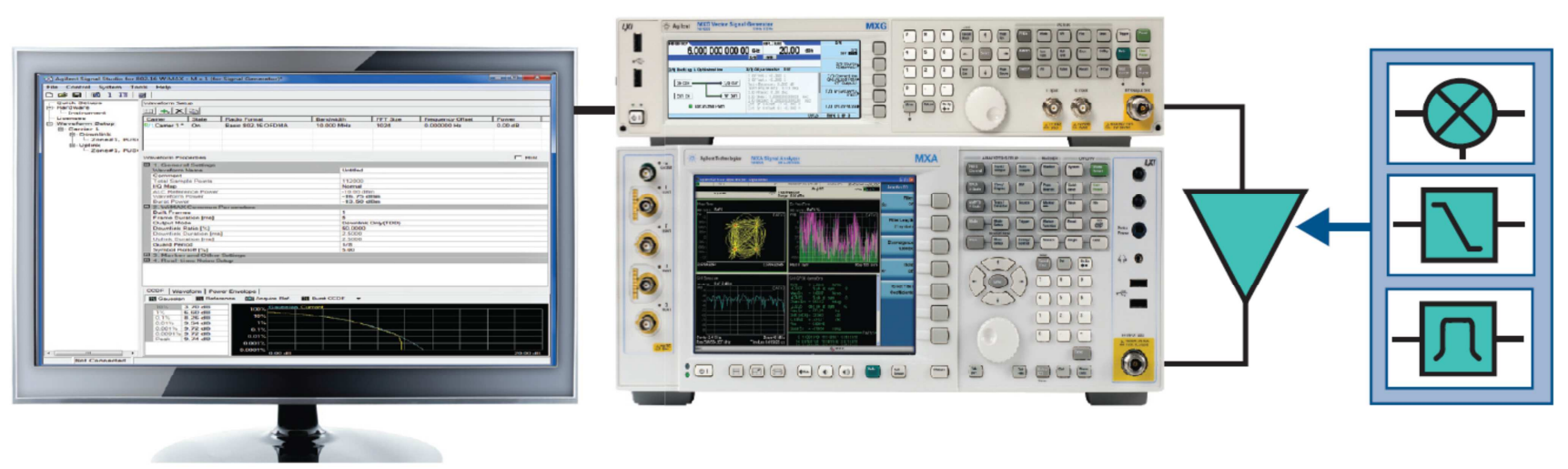

Figure 3. Component test configuration [9].

\section{Mathematics Behind}

As mentioned in mathematical theories, expressed by [10] and [11], in probability theory and statistics, the cumulative distribution function (CDF), or just distribution function, describes the probability that a real-valued random variable $X$ with a given probability distribution will be found to have a value less than or equal to $x$. In the case of a continuous distribution, it gives the area under the probability density function from minus infinity to $x$. Cumulative distribution functions are also used to specify the distribution of multivariate random variables.

The cumulative distribution function of a real-valued random variable $X$ is the function given by (1):

$$
F_{X}(x)=P(X \leq x)
$$

where the right-hand side represents the probability that the random variable $X$ takes on a value less than or equal to $x$. The probability that $X$ lies in the semi-closed interval $(a, b]$, where $a<b$, is expressed by (2):

$$
P(a<X \leq b)=F_{X}(b)-F_{X}(a)
$$

The CDF of a continuous random variable $X$ can be expressed as the integral of its probability density function $f_{X}$ as in (3):

$$
F_{X}(x)=\int_{-\infty}^{x} f_{X}(t) d t
$$

In the case of a random variable $X$ which has distribution having a discrete component at a value $b$, stated by (4),

$$
P(X=b)=F_{X}(b)-\lim _{x \rightarrow b^{-}} F_{X}(x)
$$

If $F_{X}$ is continuous at $b$, this equals zero and there is no discrete component at $b$.

Sometimes, it is useful to study the opposite question and ask how often the random variable is above a particular level. This is called the Complementary Cumulative Distribution
Function (CCDF) or simply the tail distribution or exceedance, and is defined as in (5):

$$
\bar{F}(x)=P(X>b)=1-F(x)
$$

This has applications in statistical hypothesis testing, for example, because the one-sided p-value is the probability of observing a test statistic at least as extreme as the one observed. Thus, provided that the test statistic, $T$ has a continuous distribution, the one-sided p-value is simply given by the CCDF, for an observed value $t$ of the test statistic will be expressed by (6):

$$
p=P(T \geq t)=P(T>t)=1-F_{T}(t)
$$

In survival analysis, $\bar{F}(x)$ is called the survival function and denoted $S(x)$, while the term reliability function is common in engineering.

\section{Practical Determinations}

The objective of practical determinations involves passing the generated signal through all 802.11ac available MCS. The analysis result is the digitally modulated signals characteristics identification, based on CCDF curves observation for each generated signal. Figure 4 shows the spectrum analysis diagram for a sample 802.11ac signal.

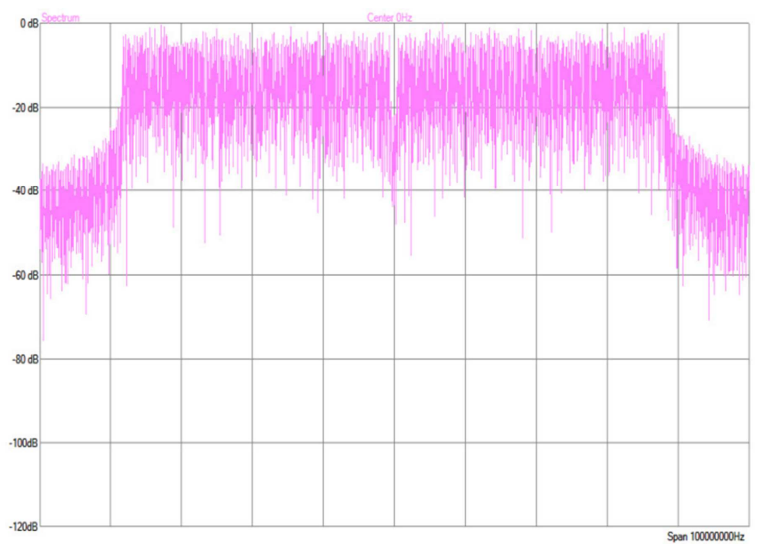

Figure 4. Signal spectrum MCS 0-9. 


\section{Results}

Diagrams from Figures 5-14 are providing an intuitive analysis of 802.11ac signals, for each specific MCS, emerged from the use of CCDF analysis functions over entire signal or only the burst part of the signal. The analysis is made by comparing the signal power with the Gaussian CCDF reference line (red line on Figs. 5-14).

To select the portion of the waveform to calculate the CCDF data the analysis charts are containing the both components available on signal design operations (Waveform CCDF and Burst CCDF, green and blue lines). Waveform CCDF will include all components of the configured frame including gaps and non-transmitted portions (both RF burst on and off portions). Burst CCDF will include the configured bursts only (this does not includes gaps or times when the RF burst is off).

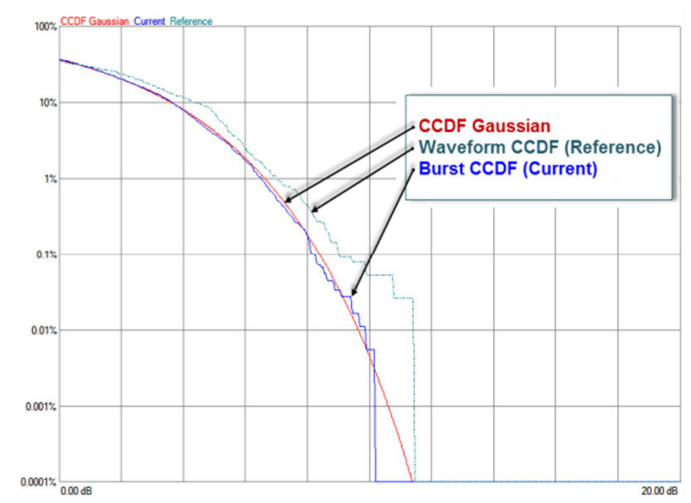

Figure 5. CCDF analysis for MCS 0 .

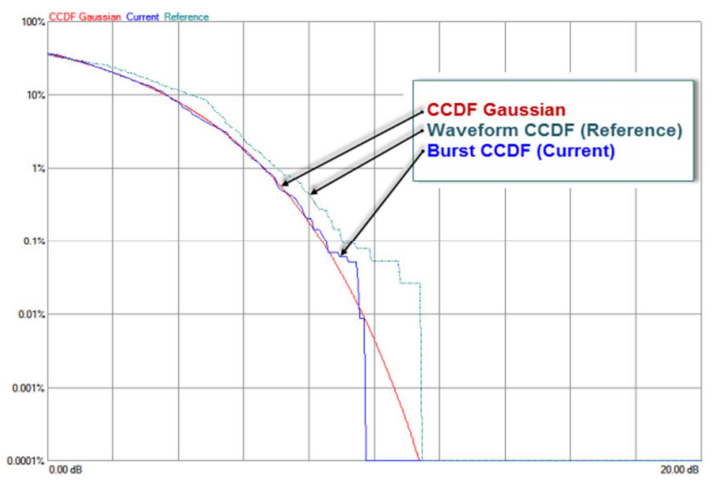

Figure 6. CCDF analysis for MCS 1 .

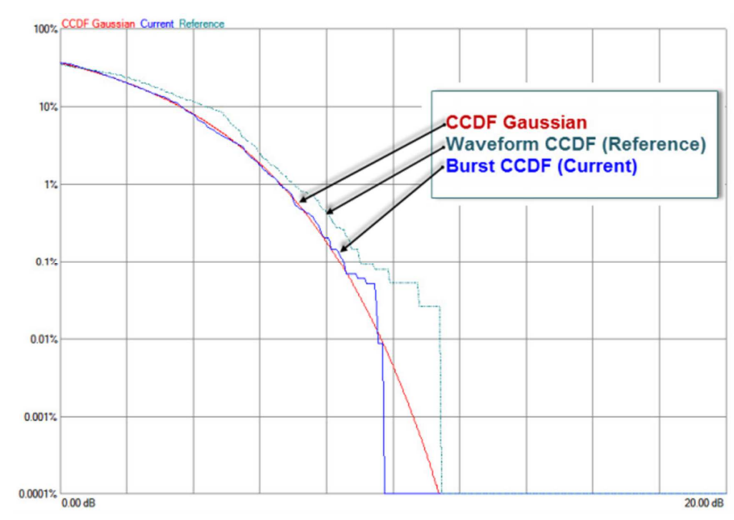

Figure 7. CCDF analysis for MCS 2.

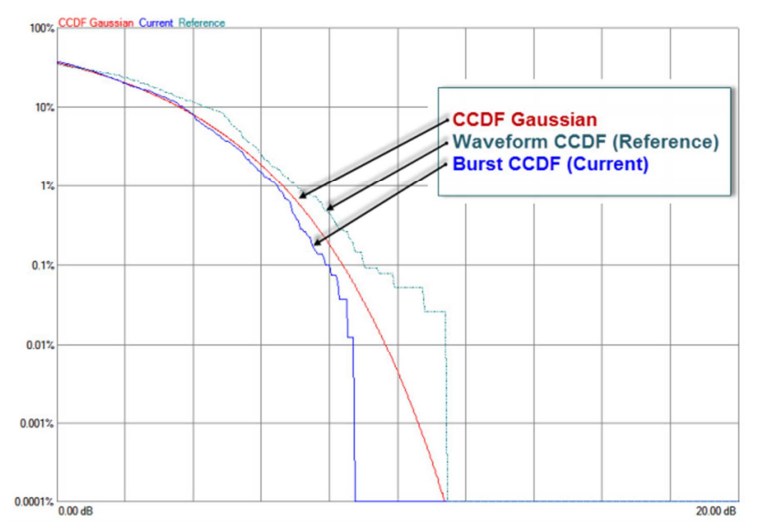

Figure 8. CCDF analysis for MCS 3 .

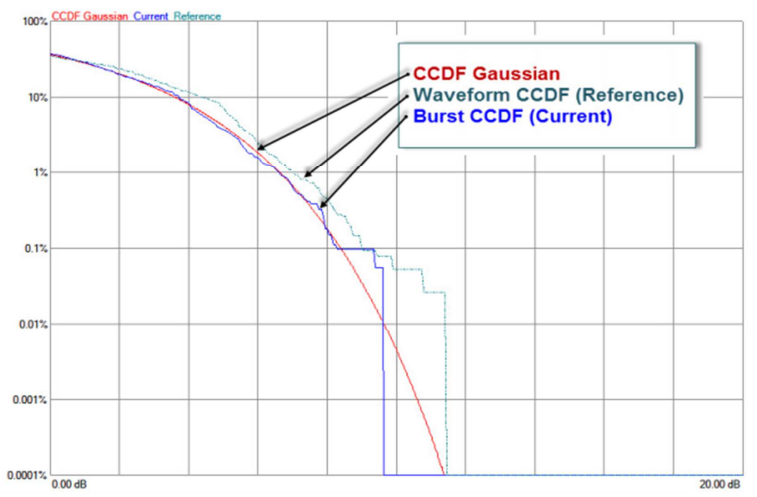

Figure 9. CCDF analysis for MCS 4.

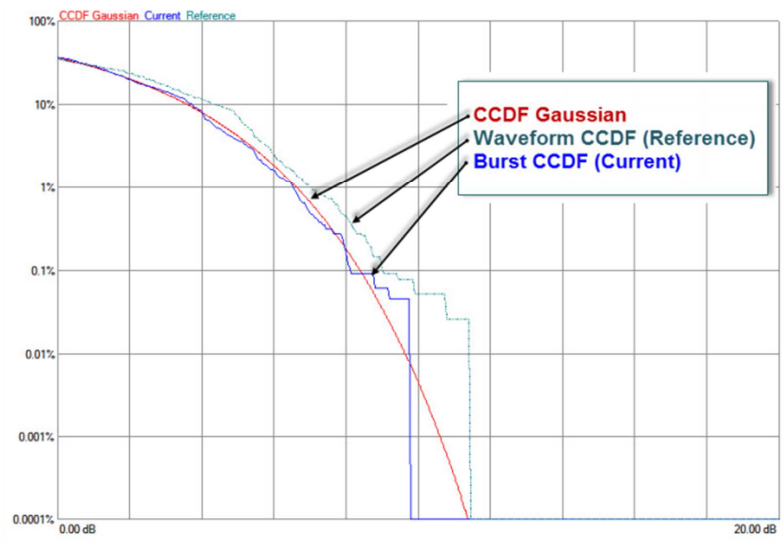

Figure 10. CCDF analysis for MCS 5.

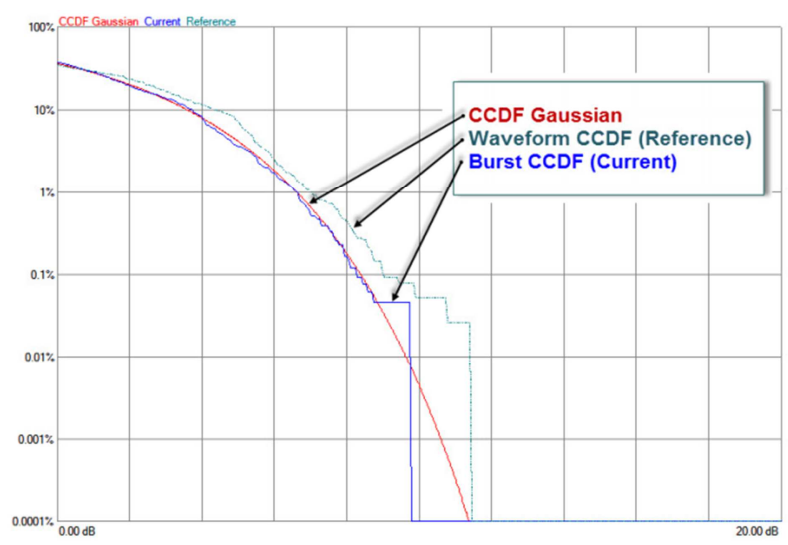

Figure 11. CCDF analysis for MCS 6. 


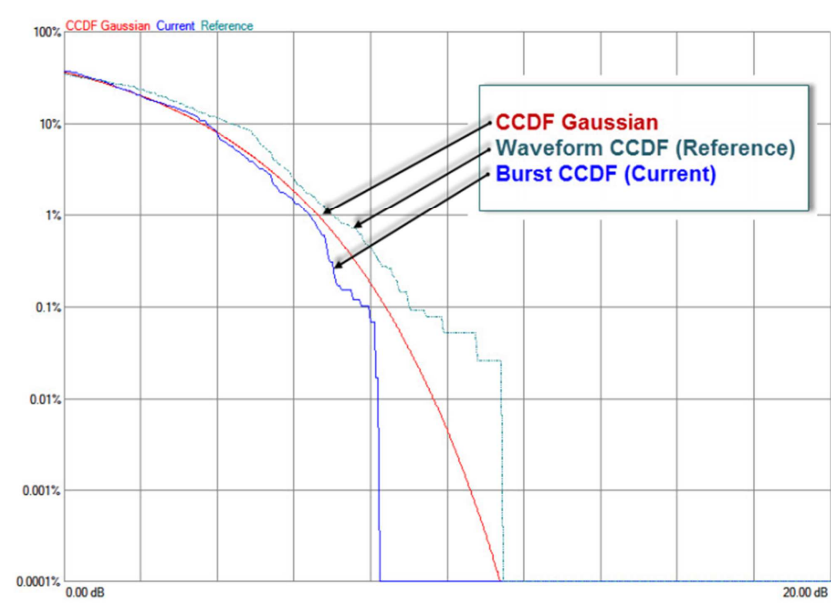

Figure 12. CCDF analysis for MCS 7.

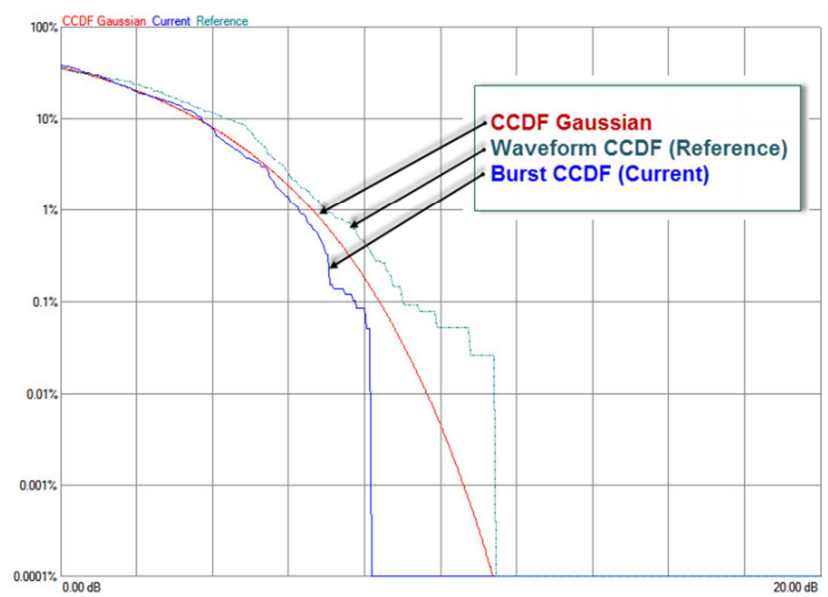

Figure 13. CCDF analysis for MCS 8 .

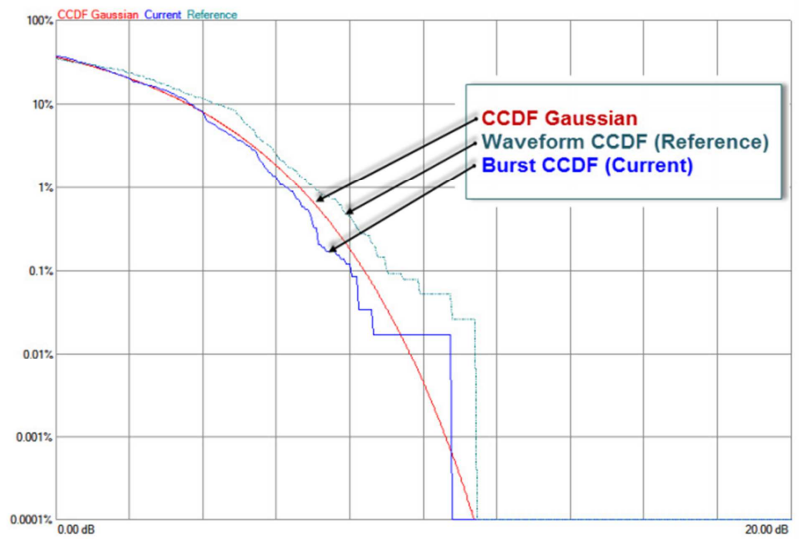

Figure 14. CCDF analysis for MCS 9.

\section{Conclusions}

It is well known that the modulation format of a signal affects its power characteristics. Using CCDF curves, we can fully characterize the power statistics of different modulation formats, and compare the results of choosing one modulation format over another.

The determinations contained in this article are guided on the behavior of radio signals in the technology 802.11ac. The outcomes obtained and presented in this paper can be used as a good base for identifying $802.11 \mathrm{ac}$ characteristics. The analysis of MCS through CCDF gives valuable information about signal levels on each available MCS on 802.11ac, taking as comparative reference the band-limited Gaussian noise reference curve.

The results presented in this study are presenting the exploration step that is a part of a started and more ample research initiative regarding wireless interference detection and prevention. Future operations are aiming the analysis of the crowded signal spectrum, faced by the wireless communications and the opportunities offered by $802.11 \mathrm{ac}$ technology to solve such problems, regarding the benefits of using multiple antenna techniques.

\section{Acknowledgements}

This paper has been financially supported within the project entitled „SOCERT. Knowledge society, dynamism through research", contract number POSDRU/159/1.5/S/132406. This project is co-financed by European Social Fund through Sectoral Operational Programme for Human Resources Development 2007-2013. Investing in people!

\section{References}

[1] IEEE $802.11 \mathrm{ac}$ http://www.ieee802.org/11/Reports/tgac/update.htm.

[2] Verma, L.; Fakharzadeh, M.; Sunghyun Choi, "Wifi on steroids: 802.11AC and 802.11AD," Wireless Communications, IEEE, vol.20, no.6, pp.30, 35, December 2013.

[3] Andrew von Nagy, "Aerohive High-Density Wi-Fi Design \& Configuration Guide v2 ", Aerohive Networks, 2013.

[4] Eng Hwee Ong; Kneckt, J.; Alanen, O.; Zheng Chang; Huovinen, T.; Nihtila, T., "IEEE 802.11ac: Enhancements for very high throughput WLANs," Personal Indoor and Mobile Radio Communications (PIMRC), 2011 IEEE 22nd International Symposium on, vol., no., pp.849,853, 11-14 Sept. 2011.

[5] Fluke Networks White Paper, Implementing 802.11ac. 2014.

[6] Agilent Application Note, Characterizing Digitally Modulated Signals with CCDF Curves, 2000.

[7] N7617B Signal Studio for WLAN 802.11a/b/g/n/ac, http://www.keysight.com/en/pd-803256-pn-N7617B/signal-stud io-for-wlan-80211a-b-g-n-ac?cc=US\&lc=eng.

[8] N5182A MXG, http://www.keysight.com/en/pd-797248-pn-N5182A/mxg-rf-ve ctor-signal-generator.

[9] Agilent Technologies, Signal Studio for WLAN 802.11a/b/g/n/ac - N7617B, Technical Overview 5990-9008EN, January 16, 2014.

[10] Cumulative distribution function, http://en.wikipedia.org/wiki/Cumulative_distribution_function

[11] Zwillinger, Daniel; Kokoska, Stephen, CRC Standard Probability and Statistics Tables and Formulae, CRC Press. p. 49. ISBN 978-1-58488-059-2, 2010. 